\title{
Exponential Stability of Uncertain T-S Fuzzy Switched Systems with Time Delay
}

\author{
Fatima Ahmida El Houssaine Tissir \\ Department of Physics, Faculty of Sciences, B.P. 1796 Fes-Atlas, Morocco
}

\begin{abstract}
This paper discusses the delay-dependent exponential stability of a class of uncertain T-S fuzzy switched systems with time delay. The method is based on Lyapunov stability theorem and free weighting matrices approach. Two illustrative examples are given to demonstrate the effectiveness of the proposed method.
\end{abstract}

Keywords: Delay dependent stability, exponential stability, linear matrix inequalities (LMIs), T-S fuzzy switched systems, uncertainty.

\section{Introduction}

A switched system belongs to an important class of hybrid systems. Typically, a switched system consists of a number of subsystems and a switching signal, which defines a specific subsystem being activated during a certain time interval. Switched systems arised in many practical models in manufacturing, communication networks, automotive engine control, chemical processes etc. ${ }^{[1-3]}$ In the last two decades, there has been increasing interest in stability analysis and control design of switched linear systems ${ }^{[4-7]}$. The main approach for stability analysis relies on the use of Lyapunov-Krasovskii functional and linear matrix inequalities (LMIs).

For nonlinear systems, there has been no systematic tool capable of finding necessary and sufficient conditions for stability till now. This makes the control problem of nonlinear systems a challenging task. The problem becomes more challenging when the system is constituted by several subsystems, and only one of them is activated at a given time because the determination of the switching rule between subsystems is a fundamental task in the stability analysis.

Fuzzy control represents an important approach to handle complex and ill-determined systems. Compared with conventional modeling techniques which use a single model to describe the global behavior of a system, fuzzy modeling is essentially a multi-model approach in which simple submodels are fuzzily combined to describe the global behavior of a system. Numerous control problems have been studied based on this T-S fuzzy model ${ }^{[8-11]}$. The fuzzy logic approach is used to study the stability analysis of switched systems $^{[8,12-14]}$.

As is well known, the introduction of time delay systems render the stability analysis more complicated. Furthermore, time delay systems are of great importance since they are frequently encountered in various engineering systems, such as chemical processes, mechanical systems, systems in economy, transmission lines, etc. Linear time delay switched systems have been extensively studied in the literatures $^{[15-20]}$. For nonlinear switched time delay systems there are only few results ${ }^{[21-24]}$. Benzaouia ${ }^{[14]}$ considered discrete time systems while Yong et al. ${ }^{[25]}$ investigated continuous time systems with constant delay.

In this paper, we address the problem of stability of un- certain switched nonlinear systems with time delay using T-S fuzzy model. Note here that the transient process of a system can be better described if its decay rate is determined. Hence, the problem of exponential stability is of great importance. To the best of our knowledge, there is no paper dealing with exponential stability of nonlinear time delay switched systems. This point represents our objective in this paper. Delay dependent exponential stability conditions are presented by using T-S fuzzy model. Our method is based on Lyapunov-Krasovskii functional approach, and the results are expressed in terms of LMIs. With our results, we can compute simultaneously the two bounds that characterize the exponential stability. These bounds are the stability factor and decay rate of the solution. Two numerical examples are given for illustration.

\section{Problem formulation}

The switched nonlinear systems with time delay can be described by a differential equation as

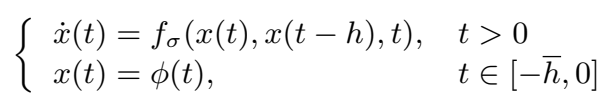

where $x \in \mathbf{R}^{n}$ is the state vector, $\phi \in\left([-\bar{h}, 0], \mathbf{R}^{n}\right)$ is the initial function, with norm $\|\phi\|=\sup _{\theta \in[-\bar{h}, 0]}\|\phi(\theta)\|$, where $\theta \in[\bar{h}, 0], f_{\sigma}$ are sufficiently regular functions from $\mathbf{R}^{n}$ to $\mathbf{R}^{n}$ that are parameterized by the index set $I:=$ $\{1,2, \cdots, N\}$, i.e., $\sigma \in I . \quad \sigma(x): \mathbf{R}^{n} \rightarrow I$ is the switching rule which is a piece-wise constant function depending on the state $x(t)$ in each time, i.e., $f_{\sigma(x)}(x(t), x(t-$ $h, t)$ switches between $f_{1}(x(t), x(t-h), t), f_{2}(x(t), x(t-$ $h), t), \cdots$, and $f_{N}(x(t), x(t-h), t) . \quad h$ is a constant time delay which can take its values in $[-\bar{h}, 0]$, i.e.,

$$
0 \leqslant h \leqslant \bar{h} \text {. }
$$

Moreover $\sigma(x)=j$ implies that the $j$-th subsystem is activated, and we have the following subsystem:

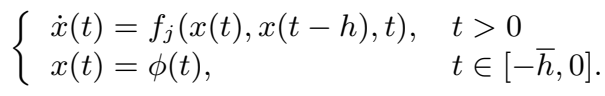

Furthermore, following the idea of [26], we consider a fuzzy switched time delay system described by T-S fuzzy model for switched nonlinear systems (1). The $i$-th rule of 
the T-S fuzzy model is described by the following IF-THEN form:

Plan rule $i$ :

$R^{i}$ : If $z_{1}(t)$ is $W_{1}^{i}$ and $\cdots$ and $z_{s}(t)$ is $W_{s}^{i}$, then

$$
\left\{\begin{aligned}
\dot{x}(t)= & \left(A_{i \sigma}+\Delta A_{i \sigma}(t)\right) x(t)+\left(B_{i \sigma}(t)+\right. \\
& \left.\Delta B_{i \sigma}(t)+\Delta B_{i \sigma}\right) x(t-h) \\
x(t)= & \phi(t), \quad t \in[-\bar{h}, 0], \quad i=1, \cdots, r \quad t>0
\end{aligned}\right.
$$

where $z_{1}(t), z_{2}(t), \cdots, z_{s}(t)$ are the premise variables, $W_{l}^{i}, l=1, \cdots, s$ are the fuzzy sets, $r$ is the number of IF-THEN rules, $\left[\begin{array}{llll}A_{i \sigma} & B_{i \sigma} & \Delta A_{i \sigma}(t) & \Delta B_{i \sigma(t)}\end{array}\right] \in$ $\left\{\left[A_{i j}, \quad B_{i j} \Delta A_{i j}(t) \Delta B_{i j}(t)\right], j=1, \cdots, N\right\}, A_{i j}$ and $B_{i j}$ are constant matrices, $\Delta A_{i j}(t)$ and $\Delta B_{i j}(t)$ are unknown perturbation matrices. The matrix $A_{i \sigma}$ switches between matrices $A_{i 1}, A_{i 2}, \cdots, A_{i N}$, the matrix $B_{i \sigma}$ switches between matrices $B_{i 1}, B_{i 2}, \cdots, B_{i N}$. The perturbation matrices $\Delta A_{i \sigma}(t)$ and $\Delta B_{i \sigma}(t)$ switch between matrices $\Delta A_{i 1}(t) A_{i 2}(t), \cdots, A_{i N}(t)$ and $\Delta B_{i 1}(t), \Delta B_{i 2}(t), \cdots, \Delta B_{i N}(t)$, respectively. Hence, system (4) can be represented as

Plan rule $i$ for individual subsystem $j$ :

$R^{i}$ : If $z_{1}(t)$ is $W_{1}^{i}$ and $\cdots$ and $z_{s}(t)$ is $W_{s}^{i}$, then

$$
\left\{\begin{array}{l}
\dot{x}(t)=\left(A_{i j}+\Delta A_{i j}(t)\right) x(t)+\left(B_{i j}(t)+\Delta B_{i j}\right) x(t-h) \\
x(t)=\phi(t), \quad t \in[-\bar{h}, 0], \quad i=1, \cdots, r, \quad t>0 .
\end{array}\right.
$$

The perturbation matrices are assumed to be norm bounded and given by

$$
\begin{aligned}
& A_{i j}=E_{a i j} F_{a i j}(t) H_{a i j} \\
& \Delta B_{i j}=E_{b i j} F_{b i j}(t) H_{b i j}
\end{aligned}
$$

where $E_{a i j}, E_{b i j}, H_{a i j}$ and $H_{b i j}$ are known constant matrices with appropriate dimensions, $F_{a i j}(t)$ and $F_{b i j}(t)$ are unknown time-varying matrices with lebesgue measurable elements bounded by

$$
\begin{aligned}
& F_{a i j}^{\mathrm{T}}(t) F_{a i j}(t) \leqslant I \\
& F_{b i j}^{\mathrm{T}}(t) F_{b i j}(t) \leqslant I .
\end{aligned}
$$

By using the center-average defuzzifier, product inference and singleton fuzzifier, dynamics of T-S fuzzy system (5) can be expressed as

$$
\begin{aligned}
\dot{x}(t)= & \sum_{i=1}^{r} \mu_{i}(z(t))\left[\left(A_{i j}+\Delta A_{i j}(t)\right) x(t)+\left(B_{i j}+\right.\right. \\
& \left.\left.\Delta B_{i j}(t)\right) x(t-h)\right]
\end{aligned}
$$

where $\mu_{i}(z(t))=\frac{w_{i}(z(t))}{\sum_{i=1}^{r} w_{i}(z(t))}, w_{i}(z(t))=\Pi_{j=1}^{s} w_{j}^{i}\left(z_{i}(t)\right)$, $W_{j}^{i}\left(z_{j}(t)\right)$ is the membership value of $z_{j}(\mathrm{t})$ in $W_{j}^{i}$, and it is assumed that $\mu_{i}(z(t)) \geqslant 0, \sum_{i=1}^{r} \mu_{i}(z(t))=1$ for all $t$.

The system (8) may be written as

$$
\dot{x}(t)=\tilde{A}_{j} x(t)+\tilde{B}_{j} x(t-h)
$$

where

$$
\begin{aligned}
& \tilde{A}_{j}=\sum_{i=1}^{r} \mu_{i}(z(t))\left(A_{i j}(t)+\Delta A_{i j}(t)\right) \\
& \tilde{B}_{j}=\sum_{i=1}^{r} \mu_{i}(z(t))\left(B_{i j}(t)+\Delta B_{i j}(t)\right) .
\end{aligned}
$$

Lemma 1. For any $x, y \in \mathbf{R}^{n}$, and $M>0$, we have

$$
2 x^{\mathrm{T}} y \leqslant x^{\mathrm{T}} M^{-1} x+y^{\mathrm{T}} M y \text {. }
$$

Definition 1. Given $\alpha>0$, system (9) is robustly $\alpha$ exponentially stable, if there exists a switching rule $\sigma$ and a constant $\beta \geqslant 1$ such that every solution $x(t, \phi)$ of the system satisfies

$$
\|x(t, \phi)\| \leqslant \beta \mathrm{e}^{-\alpha t}\|\phi\|, \quad t \geqslant 0 .
$$

\section{Main results}

Let the matrices $R, Q$ and $\tilde{Q}$ be given by

$$
\begin{aligned}
R & =\left[\begin{array}{ll}
R_{11} & R_{12} \\
R_{12}^{\mathrm{T}} & R_{22}
\end{array}\right] \\
Q & =\left[\begin{array}{ll}
Q_{11} & Q_{12} \\
Q_{12}^{\mathrm{T}} & Q_{22}
\end{array}\right] \\
\tilde{Q} & =\left[\begin{array}{ll}
I & A_{i j}^{\mathrm{T}} \\
0 & B_{i j}^{\mathrm{T}}
\end{array}\right] Q\left[\begin{array}{ll}
I & 0 \\
A_{i j} & B_{i j}
\end{array}\right] .
\end{aligned}
$$

And let

$$
\begin{aligned}
& \bar{Q}=\left[\begin{array}{ll}
0 & E_{a i j}^{\mathrm{T}} \\
0 & E_{b i j}^{\mathrm{T}}
\end{array}\right] \\
& Q=\left[\begin{array}{cc}
0 & 0 \\
E_{a i j} & E_{b i j}
\end{array}\right] \\
& \bar{H}_{i j}=\left[\begin{array}{ll}
H_{a i j} & 0 \\
0 & H_{b i j}
\end{array}\right] \\
& \beta_{1}=\lambda_{\min }\left(R_{11}\right)
\end{aligned}
$$

$$
\begin{gathered}
\beta_{2}=\left(1+\bar{h}^{2}\right) \lambda_{\max }(R)+\frac{1-\mathrm{e}^{2 \alpha \bar{h}}}{2 \alpha} \lambda_{\max }(P)+ \\
\left.\frac{\mathrm{e}^{2 \alpha \bar{h}}+2 \alpha \bar{h}-1}{\alpha^{2}} \max _{\substack{1 \leqslant i \leqslant r \\
1 \leqslant j \leqslant N}}\left(\tilde{Q}_{i j}\right)+\lambda_{\max }\left(\bar{H}_{i j}^{\mathrm{T}} \bar{H}_{i j}\right)\right\}
\end{gathered}
$$$$
L_{i j}=R_{11} A_{i j}+A_{i j}^{\mathrm{T}} R_{11}+F_{0} A_{i j}+A_{i j}^{\mathrm{T}} F_{0}^{\mathrm{T}}+Z
$$$$
Y_{i j}=\left[\begin{array}{cc}
\left(F_{0}+R_{11}\right) E_{a i j} & \left(F_{0}+R_{11}\right) E_{b i j} \\
F_{1} E_{a i j} & F_{1} E_{b i j} \\
\left(F_{2}+R_{12}^{\mathrm{T}}\right) E_{a i j} & \left(F_{2}+R_{12}^{\mathrm{T}}\right) E_{b i j} \\
F_{3} E_{a i j} & F_{3} E_{b i j}
\end{array}\right]
$$

$$
N_{i j}=\left[\begin{array}{cccc}
H_{a i j}^{\mathrm{T}} S_{0 i j} H_{a i j} & * & * & * \\
H_{b i j}^{\mathrm{T}} S_{1 i j}^{\mathrm{T}} H_{a i j} & H_{a i j}^{\mathrm{T}} S_{2 i j} H_{b i j} & * & * \\
0 & 0 & 0 & * \\
0 & 0 & 0 & 0
\end{array}\right]
$$

$$
\begin{gathered}
S_{i j}=\left(\begin{array}{cc}
S_{0 i j} & S_{1 i j} \\
S_{1 i j}^{\mathrm{T}} & S_{2 i j}
\end{array}\right) \\
\pi_{i j}=\left[\begin{array}{cccc}
0 & * & * & * \\
F_{1} A_{i j}+B_{i j}^{\mathrm{T}} & F_{1} B_{i j}+B_{i j}^{\mathrm{T}} & * & * \\
F_{2} A_{i j} & F_{2} B_{i j} & 0 & * \\
F_{3} A_{i j}-F_{0}^{\mathrm{T}} & F_{3} B_{i j}-F_{1}^{\mathrm{T}} & F_{2}^{\mathrm{T}} & -F_{3}-F_{3}^{\mathrm{T}}
\end{array}\right]
\end{gathered}
$$

$$
\psi_{i j}=\left[\begin{array}{cccc}
\psi_{11}^{i j} & * & * & * \\
\psi_{21}^{i j} & \psi_{22}^{i j} & * & * \\
\psi_{31}^{i j} & \psi_{32}^{i j} & \psi_{33}^{i j} & * \\
\bar{h} Q_{12}^{\mathrm{T}} & 0 & 0 & \bar{h} Q_{22}
\end{array}\right]
$$

where ${ }^{*}$ denotes the symmetric part in a symmetric matrix, and

$$
\psi_{11}^{i j}=R_{12}+R_{12}^{\mathrm{T}}+2 \alpha R_{11}+P-Z-\frac{1}{\bar{h}} \mathrm{e}^{-2 \alpha \bar{h}} Q_{22}+\bar{h} Q_{11}
$$




$$
\begin{aligned}
& \psi_{21}^{i j}=B_{i j}^{\mathrm{T}} R_{11}-R_{12}^{\mathrm{T}}+\frac{1}{\bar{h}} \mathrm{e}^{-2 \alpha \bar{h}} Q_{22} \\
& \psi_{31}^{i j}=R_{12}^{\mathrm{T}} A_{i j}+R_{22}+2 \alpha R_{12}^{\mathrm{T}}-\frac{1}{\bar{h}} \mathrm{e}^{-2 \alpha \bar{h}} Q_{12} \\
& \psi_{22}^{i j}=-\mathrm{e}^{-2 \alpha \bar{h}} P-\frac{1}{\bar{h}} \mathrm{e}^{-2 \alpha \bar{h}} Q_{22} \\
& \psi_{32}^{i j}=R_{12}^{\mathrm{T}} B_{i j}-R_{22}+\frac{1}{\bar{h}} \mathrm{e}^{-2 \alpha \bar{h}} Q_{12} \\
& \psi_{33}^{i j}=2 \alpha R_{22}-\frac{1}{\bar{h}} \mathrm{e}^{-2 \alpha \bar{h}} Q_{11} .
\end{aligned}
$$

The switching rule $\left(\left[S_{R}\right]\right)$ is constructed as follows:

Step 1. Let $x(t)=\phi(t)$.

Step 2. $\sigma(x)=\arg \min _{1 \leqslant j \leqslant N}\left\{x^{\mathrm{T}}(t)\left[\left(\sum_{i=1}^{r} \mu_{i} A_{i j}\right)^{\mathrm{T}} \times\right.\right.$ $\left.\left.\left(R_{11}+F_{0}^{\mathrm{T}}\right)+\left(R_{11}+F_{0}\right)\left(\sum_{i=1}^{r} \mu_{i} A_{i j}\right)+Z\right] x(t)\right\}$.

Step 3. $\sigma(x)=i$ as long as $x(t) \in \Omega_{i}=$ $\left\{x: x^{\mathrm{T}}(t)\left[\left(\sum_{i=1}^{r} \mu_{i} \times A_{i j}\right)^{\mathrm{T}}\left(R_{11}+F_{0}^{\mathrm{T}}\right)+\left(R_{11}+F_{0}\right) \times\right.\right.$ $\left.\left.\left(\sum_{i=1}^{r} \mu A_{i j}\right)+Z\right] x(t)<0\right\}$.

Step 4. If $x(t)$ hits the boundary of $\Omega_{i}$, go to Step 2 to determine the next mode.

Theorem 1. System (9) is $\alpha$-exponentially stable if there exist symmetric positive definite matrices $Z, P, S$, $Q_{22}, Q_{11}, R_{11}, R_{22}, S_{0 i j}, S_{2 i j}$, matrices $R_{12}, Q_{12}, F_{0}, F_{1}$, $F_{2}, F_{3}, S_{1 i j}, j=1, \cdots, N$ and $i=1, \cdots, r$ satisfying:

1) There exists $0<\rho_{j}<1$, such that $\sum_{j=1}^{N} \rho_{j}=1$ and

$$
\sum_{j=1}^{N} \rho_{j} L_{i j}<0
$$

2)

$$
\left[\begin{array}{cc}
\psi_{i j}+\pi_{i j}+N_{i j} & * \\
Y_{i j}^{\mathrm{T}} & -S_{i j}
\end{array}\right]<0
$$

The switching rule is given by $\left[S_{R}\right]$, and the solution $x(t, \phi)$ of the system satisfies

$$
\|x(t, \phi)\| \leqslant \beta \mathrm{e}^{-\alpha t}\|\phi\|, \quad \beta=\sqrt{\frac{\beta_{2}}{\beta_{1}}}, \quad t \geqslant 0 .
$$

Proof. Let $x_{t}:=\{x(t+s), s \in[-2 \bar{h}, 0]\}$ and consider the following Lyapunov-Krasovskii functional: $V\left(x_{t}\right)=$ $V_{1}\left(x_{t}\right)+V_{2}\left(x_{t}\right)+V_{3}\left(x_{t}\right)$, where

$$
\begin{gathered}
V_{1}\left(x_{t}\right)=\left(\begin{array}{c}
x(t) \\
\int_{t-h}^{\mathrm{T}} x(s) \mathrm{d} s
\end{array}\right)^{\mathrm{T}} R\left(\begin{array}{c}
x(t) \\
\int_{t-h}^{\mathrm{T}} x(s) \mathrm{d} s
\end{array}\right)^{\mathrm{T}} \\
V_{2}\left(x_{t}\right)=\int_{-h}^{0} \mathrm{e}^{2 a \theta} s x^{\mathrm{T}}(t+s) P x(t+s) \mathrm{d} s \\
V_{3}\left(x_{t}\right)=\int_{-h}^{0} \int_{s}^{0} \mathrm{e}^{2 a \theta}\left(\begin{array}{c}
x(t+\theta) \\
\dot{x}(t+\theta)
\end{array}\right)^{\mathrm{T}} Q\left(\begin{array}{c}
x(t+\theta) \\
\left.\dot{x}_{t+\theta}\right)
\end{array}\right) \mathrm{d} \theta \mathrm{d} s .
\end{gathered}
$$

It is easy to verify that

$$
\begin{aligned}
& V_{1}\left(x_{t}\right) \leqslant\left(1+\bar{h}^{2}\right) \lambda_{\max }(R)\left\|x_{t}\right\|^{2} \\
& V_{2}\left(x_{t}\right) \leqslant \frac{1-\mathrm{e}^{-2 \alpha \bar{h}}}{2 \alpha} \lambda_{\max }(P)\left\|x_{t}\right\|^{2} \\
& V_{3}\left(x_{t}\right)=\int_{-h}^{0} \int_{s}^{0} \mathrm{e}^{2} \alpha \theta\left(\begin{array}{c}
x(t+\theta) \\
x(t+\theta-h(t))
\end{array}\right)^{\mathrm{T}} \times \\
& {\left[\begin{array}{cc}
I & \tilde{A}_{i j}^{\mathrm{T}} \\
0 & \tilde{B}_{i j}^{\mathrm{T}}
\end{array}\right] Q\left[\begin{array}{cc}
I & 0 \\
\tilde{A}_{i j} & \tilde{B}_{i j}
\end{array}\right]\left(\begin{array}{c}
x(t+\theta) \\
x(t+\theta-h)
\end{array}\right) \mathrm{d} \theta \mathrm{d} s \leqslant}
\end{aligned}
$$

$$
\begin{aligned}
& \int_{-h}^{0} \int_{s}^{0} \mathrm{e}^{2 \alpha \theta}\left(\begin{array}{c}
x(t+\theta) \\
x(t+\theta-h)
\end{array}\right)^{\mathrm{T}} \times \\
& {\left[2 \tilde{Q}_{i j}+2 \tilde{H}_{i j}^{\mathrm{T}} \bar{F}_{i j}^{\mathrm{T}} \bar{Q}_{i j} \bar{F}_{i j} \bar{F}_{i j}\right]\left(\begin{array}{c}
x(t+\theta) \\
x(t+\theta-h)
\end{array}\right) \mathrm{d} \theta \mathrm{d} s \leqslant} \\
& \frac{\mathrm{e}^{-2 \alpha \bar{h}}+2 \alpha \bar{h}-1}{\alpha^{2}} \max _{\substack{1 \leqslant i \leqslant s \\
1 \leqslant j \leqslant N}}\left\{\lambda_{\max }\left(\tilde{Q}_{i j}\right)+\right.
\end{aligned}
$$$$
\left.\lambda_{\max }\left(\bar{Q}_{i j}\right) \lambda_{\max }\left(\bar{H}_{i j}^{\mathrm{T}} \bar{H}_{i j}\right)\right\}
$$

where $\bar{F}_{i j}=\operatorname{diag}\left\{F_{a i j}, F_{b i j}\right\}$. So we have

$$
\beta_{1}\|x(t)\|^{2} \leqslant V\left(x_{t}\right) \leqslant \beta_{2}\left\|x_{t}\right\|^{2}
$$

where $\beta_{1}$ and $\beta_{2}$ are defined by (12) and (13). Computing the first time derivative of $V\left(x_{t}\right)$, we obtain

$$
\begin{aligned}
& \dot{V}_{1}\left(x_{t}\right)=2\left(\begin{array}{c}
x(t) \\
\int_{t-h}^{t} x(s) \mathrm{d} s
\end{array}\right)^{\mathrm{T}} R\left(\begin{array}{c}
\dot{x}(t) \\
x(t)-x(t-h)
\end{array}\right) \\
& \dot{V}_{2}\left(x_{t}\right)=x^{\mathrm{T}}(t) P x(t)-2 \alpha V_{2}\left(x_{t}\right)-\mathrm{e}^{-2 \alpha h} x^{\mathrm{T}}(t-h) P x(t-h) \\
& \dot{V}_{3}\left(x_{t}\right)=h\left(\begin{array}{c}
x(t) \\
\dot{t}(t)
\end{array}\right)^{\mathrm{T}} Q\left(\begin{array}{c}
x(t) \\
\dot{(}(t)
\end{array}\right)-2 \alpha V_{3}\left(x_{t}\right)- \\
& \int_{t-h}^{t} \mathrm{e}^{-2 \alpha(s-t)}\left(\begin{array}{c}
x(s) \\
\dot{x}(s)
\end{array}\right)^{\mathrm{T}} Q\left(\begin{array}{c}
x(s) \\
\dot{x}(s)
\end{array}\right) \mathrm{d} s . \\
& \text { Let } \quad \chi(t)=\left(\begin{array}{c}
x(t) \\
x(t-h) \\
\int_{t-h}^{t} x(s) \mathrm{d} s \\
\dot{x}(t)
\end{array}\right) \text {, then } \\
& \dot{V}_{1}\left(x_{t}\right)=\sum_{i=1}^{r} \mu_{i}\left[x^{\mathrm{T}}(t)\left(R_{11} A_{i j}+A_{i j}^{\mathrm{T}} R_{11}\right) x(t)+\right. \\
& \left.\chi^{\mathrm{T}}(t)\left(T_{1}+\Delta T_{1}\right) \chi(t)\right]-2 \alpha V_{1}(t) \\
& \dot{V}_{2}\left(x_{t}\right)=\chi^{\mathrm{T}}(t) T_{2} P T_{2}^{\mathrm{T}} \chi(t)-\chi^{\mathrm{T}}(t) T_{3} P T_{3}^{\mathrm{T}} \chi(t)-2 \alpha V_{2}(t) \\
& \dot{V}_{3}\left(x_{t}\right)=\chi^{\mathrm{T}}(t) T_{4} \chi(t)-2 \alpha V_{3}(t)
\end{aligned}
$$

where

$T_{1}=$

$$
\left[\begin{array}{cccc}
R_{12}+R_{12}^{\mathrm{T}}+2 \alpha R_{11} & * & * & * \\
B_{i j}^{\mathrm{T}} R_{11}-R_{12}^{\mathrm{T}} & 0 & * & * \\
R_{12}^{\mathrm{T}} A_{i j}+R_{22}+2 \alpha R_{12}^{\mathrm{T}} & R_{12}^{\mathrm{T}} B_{i j}-R_{22} & 2 \alpha R_{22} & * \\
0 & 0 & 0 & 0
\end{array}\right]
$$$$
T_{4}=
$$$$
\left[\begin{array}{cccc}
h Q_{11}-\frac{1}{h} \mathrm{e}^{-2 \alpha \bar{h}} Q_{22} & * & * & * \\
\frac{1}{h} \mathrm{e}^{-2 \alpha \bar{h}} Q_{22} & -\frac{1}{h} \mathrm{e}^{-2 \alpha \bar{h}} Q_{22} & * & * \\
-\frac{1}{h} \mathrm{e}^{-2 \alpha \bar{h}} Q_{12} & \frac{1}{h} \mathrm{e}^{-2 \alpha \bar{h}} Q_{12} & -\frac{1}{h} \mathrm{e}^{-2 \alpha \bar{h}} Q_{11} & * \\
h Q_{12}^{\mathrm{T}} & 0 & 0 & h Q_{22}
\end{array}\right]
$$$$
\Delta T_{1}=\left[\begin{array}{cccc}
R_{11} \Delta A_{i j}+\Delta A_{i j}^{\mathrm{T}} R_{11} & * & * & * \\
\Delta B_{i j}^{\mathrm{T}} R_{11} & 0 & * & * \\
R_{12}^{\mathrm{T}} \Delta A_{i j} & R_{12}^{\mathrm{T}} \Delta B_{i j} & 0 & * \\
0 & 0 & 0 & 0
\end{array}\right]
$$

$T_{2}=\left[\begin{array}{l}I \\ 0 \\ 0 \\ 0\end{array}\right]$ 


$$
T_{3}=\left[\begin{array}{c}
0 \\
\mathrm{e}^{-\alpha h} I \\
0 \\
0
\end{array}\right]
$$

Let $\theta_{i j}=T_{1}+T_{2} P T_{2}^{\mathrm{T}}-T_{3} P T_{3}^{\mathrm{T}}+T_{4}$, we get

$$
\begin{gathered}
\dot{V}\left(x_{t}\right)+2 \alpha V\left(x_{t}\right) \leqslant \sum_{i=1}^{r} \mu_{i}\left[x ^ { \mathrm { T } } ( t ) \left(R_{11} A_{i j}+\right.\right. \\
\left.\left.A_{i j}^{\mathrm{T}} R_{11}\right) x(t)\right]+\sum_{i=1}^{r} \mu_{i}\left[\chi^{\mathrm{T}}(t) \theta_{i j} \chi(t)+\right. \\
\left.\chi^{\mathrm{T}}(t) \Delta T_{1} \chi(t)\right] .
\end{gathered}
$$

Now let $B_{j}=\left[\begin{array}{llll}\tilde{A}_{j} & \tilde{B}_{j} & 0 & -I\end{array}\right]$ and $F=\left[\begin{array}{c}F_{0} \\ F_{1} \\ F_{2} \\ F_{3}\end{array}\right]$, we can easily verify that $B_{j} \chi=0, \forall \chi \neq 0$, and

$$
\chi^{\mathrm{T}}(t)\left[F B_{j}+B_{j}^{\mathrm{T}} F^{\mathrm{T}}\right] \chi(t)=0, \quad j=1, \cdots, N .
$$

Adding (19) to the right hand side of (18), and adding and subtracting the term $x^{\mathrm{T}}(t) Z x(t)$ with the positive definite matrix $Z$, we get

$$
\begin{gathered}
\dot{V}\left(x_{t}\right)+2 \alpha V\left(x_{t}\right) \leqslant \sum_{i=1}^{r} \mu_{i}\left[x^{\mathrm{T}}(t) L_{i j} x(t)\right]+\sum_{i=1}^{r} \mu_{i} \times \\
{\left[\chi^{\mathrm{T}}(t)\left[\theta_{i j}+\pi_{i j}\right] \chi(t)+\chi^{\mathrm{T}}(t) \Delta \pi_{i j} \chi(t)\right]}
\end{gathered}
$$

where $\Delta \pi_{i j}$ is given at the top of the next page.

$$
\begin{gathered}
\text { Let } \quad \tilde{F}=\left[\begin{array}{ccc}
F_{a i j}(t) & 0 \\
0 & F_{b i j}(t)
\end{array}\right] \text { and } \tilde{H}_{i j}= \\
{\left[\begin{array}{cccc}
H_{a i j} & 0 & 0 & 0 \\
0 & H_{b i j} & 0 & 0
\end{array}\right] . \Delta \pi_{i j} \text { can be written as }} \\
\Delta \pi_{i j}=Y_{i j} \tilde{F} \tilde{H}_{i j}+\tilde{H}_{i j}^{\mathrm{T}} \tilde{F}^{\mathrm{T}} Y_{i j}^{\mathrm{T}} .
\end{gathered}
$$

Applying Lemma 1, we have

$$
\begin{gathered}
\dot{V}\left(x_{t}\right)+2 \alpha V\left(x_{t}\right) \leqslant \sum_{i=1}^{r} \mu_{i}\left\{x^{\mathrm{T}}(t) L_{i j} x(t)+\chi^{\mathrm{T}}(t)\left[\theta_{i j}+\right.\right. \\
\left.\pi_{i j}+Y_{i j} S_{i j}^{-1} Y_{i j} T+H_{i j} T S_{i j} H_{i j}\right] \chi(t)
\end{gathered}
$$

where $\theta_{i j}$ can be written as

$$
\begin{aligned}
\theta_{i j}=M_{i j}+h & {\left[\begin{array}{cccc}
Q_{11} & * & * & * \\
0 & -\frac{\mathrm{e}^{-2 \alpha h}}{h} P & * & * \\
0 & 0 & 0 & * \\
Q_{12}^{\mathrm{T}} & 0 & 0 & Q_{22}
\end{array}\right]-} \\
& \frac{\mathrm{e}^{-2 \alpha h}}{h} \times\left[\begin{array}{cccc}
Q_{22} & * & * & * \\
-Q_{22} & Q_{22} & * & * \\
Q_{12} & -Q_{12} & Q_{11} & * \\
0 & 0 & 0 & 0
\end{array}\right]
\end{aligned}
$$

where $M_{i j}$ is given at the top of the next page.

$$
\begin{gathered}
\theta_{i j}=M_{i j}+h\left[\begin{array}{cccc}
Q_{11} & * & * & * \\
0 & \lambda(h) P & * & * \\
0 & 0 & 0 & * \\
Q_{12}^{\mathrm{T}} & 0 & 0 & Q_{22}
\end{array}\right]+ \\
\lambda(h)\left[\begin{array}{cc}
0 & -I \\
0 & I \\
I & 0 \\
0 & 0
\end{array}\right]\left[\begin{array}{lll}
Q_{12} & Q_{12} \\
Q_{12}^{\mathrm{T}} & Q_{22}
\end{array}\right]\left[\begin{array}{cccc}
0 & 0 & I & 0 \\
-I & I & 0 & 0
\end{array}\right]
\end{gathered}
$$

where $\lambda(h)=-\frac{\mathrm{e}^{-2 \alpha h}}{h}$. Since $\lambda(h)$ is an increasing function of $h$ we have $\lambda(h) \leqslant \lambda(\bar{\lambda})$ so that

$$
\begin{gathered}
\theta_{i j} \leqslant \Theta_{i j}=M_{i j}+\bar{h}\left[\begin{array}{cccc}
Q_{11} & * & * & * \\
0 & -\frac{\mathrm{e}^{-2 \alpha \bar{h}}}{\bar{h}} P & * & * \\
0 & 0 & 0 & * \\
Q_{12}^{\mathrm{T}} & 0 & 0 & Q_{22}
\end{array}\right]- \\
\frac{\mathrm{e}^{-2 \alpha \bar{h}}}{\bar{h}}\left[\begin{array}{cc}
0 & -I \\
0 & I \\
I & 0 \\
0 & 0
\end{array}\right]\left[\begin{array}{ll}
Q_{11} & Q_{12} \\
Q_{12}^{\mathrm{T}} & Q_{22}
\end{array}\right]\left[\begin{array}{cccc}
0 & 0 & I & 0 \\
-I & I & 0 & 0
\end{array}\right] .
\end{gathered}
$$

Since condition (16) holds, by schur complement, for all $h$ satisfying $0<h \leqslant \bar{h}$, we have

$$
\Theta_{i j}+\pi_{i j}+N_{i j}+Y_{i j} S_{i j}^{-1} Y_{i j}^{\mathrm{T}}<0
$$

where $N_{i j}=\tilde{H}_{i j}^{\mathrm{T}} S_{i j} \tilde{H}_{i j}$. It follows that

$$
\dot{V}\left(x_{t}\right)+2 \alpha V\left(x_{t}\right) \leqslant \sum_{i=1}^{r} \mu_{i}\left\{x^{\mathrm{T}}(t) L_{i j} x(t)\right\} .
$$

From condition (15), we have $\sum_{j=1}^{N} \rho_{j} L_{i j}<0$, where $0<\rho_{j}<1, j=1, \cdots, N$, and $\sum_{j=1}^{N} \rho_{j}=1$. Consequently, we can write

$$
\begin{gathered}
\sum_{j=1}^{N} \rho_{j} \min _{j=1, \cdots, N}\left(\sum_{i=1}^{r} \mu_{i}\left[x^{\mathrm{T}}(t) L_{i j} x(t)\right]\right) \leqslant \\
\sum_{j=1}^{N} \rho_{j} \sum_{i=1}^{r} \mu_{i} x^{\mathrm{T}}(t) L_{i j} x(t)<0 .
\end{gathered}
$$

By choosing the switching rule

$$
\sigma(x)=\arg \min _{j=1, \cdots, N}\left(\sum_{i=1}^{r} \mu_{i}\left[x^{\mathrm{T}}(t) L_{i j} x(t)\right]\right)
$$

we have

$$
\begin{gathered}
\dot{V}\left(x_{t}\right)+2 \alpha V\left(x_{t}\right) \leqslant \sum_{i=1}^{r} \mu_{i}\left[x^{\mathrm{T}}(t) L_{i j} x(t)\right] \leqslant \\
\sum_{j=1}^{N} \rho_{j}\left[\sum_{i=1}^{r} \mu_{i} x^{\mathrm{T}}(t) L_{i j} x(t)\right]= \\
\sum_{i=1}^{r} \mu_{j}\left[\sum_{j=1}^{N} \rho_{j} x^{\mathrm{T}}(t) L_{i j} x(t)\right]<0 .
\end{gathered}
$$

This implies that $V\left(x_{t}\right) \leqslant V(\phi) \mathrm{e}^{-2 \alpha t}, t \geqslant 0$. Taking account of (17), we obtain $\beta_{1}\|x(t)\|^{2} \leqslant V\left(x_{t}\right) \leqslant V(\phi) \mathrm{e}^{-2 \alpha t} \leqslant$ $\beta_{2} \mathrm{e}^{-2 \alpha t}\|\phi\|^{2}$. And then, $\|x(t)\| \leqslant \beta \mathrm{e}^{-\alpha t}\|\phi\|, t \geqslant 0$ which concludes the proof.

Remark 1. For the nominal system of (9), i.e., $\Delta A_{i j}(t)=\Delta B_{i j}(t)=0$, from Theorem 1 we can obtain the $\alpha$-exponential stability if the condition (15) holds and the following LMI is satisfied.

$$
\psi_{i j}+\pi_{i j}<0
$$




$$
\begin{gathered}
\Delta \pi_{i j}=\left[\begin{array}{cccc}
\left(F_{0}+R_{11}\right) \Delta A_{i j}+\Delta A_{i j}^{\mathrm{T}}\left(F_{0}^{\mathrm{T}}+R_{11}\right) & * & * & * \\
F_{1} \Delta A_{i j}+\Delta B_{i j}^{\mathrm{T}}\left(F_{0}^{\mathrm{T}}+R_{11}\right) & F_{1} \Delta B_{i j}+\Delta B_{i j}^{\mathrm{T}} F_{1}^{\mathrm{T}} & * & * \\
\left(F_{2}+R_{12}^{\mathrm{T}}\right) \Delta A_{i j} & \left(F_{2}+R_{12}\right) \Delta B_{i j} & 0 & * \\
F_{3} \Delta A_{i j} & F_{3} \Delta B_{i j} & 0 & 0
\end{array}\right] \\
M_{i j}=\left[\begin{array}{cccc}
R_{12}+R_{12}^{\mathrm{T}}+2 \alpha R_{11}+P-Z & * & * & * \\
B_{i j}^{\mathrm{T}} R_{11}-R_{12}^{\mathrm{T}} & 0 & * & * \\
R_{12}^{\mathrm{T}} A_{i j}+R_{22}+2 \alpha R_{12}^{\mathrm{T}} & R_{12}^{\mathrm{T}} B_{i j}-R_{22} & 2 \alpha R_{22} & * \\
0 & 0 & 0 & 0
\end{array}\right] .
\end{gathered}
$$

Remark 2. If we set $\alpha=0$ in the Lyapunov functional, Theorem 1 reduces to a robust stability criterion. In [27], the free weighting matrix method was used to deal with the cross terms. It has been shown that the weighting matrix approach is less restrictive than model transformation method used in [28]. Peng et al. ${ }^{[29]}$ employed Jensen's integral inequality, and it is shown by examples that it gives improved results. The work of [30] is based on Finsler's lemma and leads to improved results over the previous references. We emphasize here that the results of [27-30] can not be applied to switched systems, because the stability of the switched system depends on the switching rule. As is well known, all the subsystems may be stable, but the overall system may be unstable if the switching rule is not adequately chosen and the converse is true. In this paper, we are motivated by Finsler's lemma although this lemma is not used. In manipulating the derivative of the Lyapunov functional, we used the state equation. As shown in example 1, our approach gives improved results.

Remark 3. For the case $N=1$, without switching, and without perturbations, system (9) reduces to the following T-S fuzzy time delay system:

$$
\dot{x}(t)=\sum_{i=1}^{r} \mu_{i}\left[A_{i} x(t)+B_{i} x(t-h)\right] .
$$

In this case, from (20), since there is no switching rule, we can insert $L_{i j}$ in the first element of the matrix $\theta_{i j}$, which leads to the result stated in the next corollary. First, let $\psi^{i}=\psi^{i j}$ and $\pi_{i}=\pi_{i j}$, where in the expressions of $\psi^{i j}$ and $\pi_{i j}$, we replace $A_{i j}$ and $B_{i j}$ by $A_{i}$ and $B_{i}$, respectively.

Corollary 1. The system (24) is $\alpha$-exponentially stable if there exist symmetric positive definite matrices $P, Q_{22}$, $Q_{11}, R_{11}, R_{22}, Z_{1}, Z_{2}$, matrices $R_{12}, Q_{12}, F_{0}, F_{1}, F_{2}, F_{3}$ satisfying

$$
\Omega^{i}+\pi_{i}<0
$$

The solution $x(t, \phi)$ of the system satisfies

$$
\begin{gathered}
\|x(t, \phi)\| \leqslant \beta \mathrm{e}^{-\alpha t}\|\phi\| \\
\beta=\sqrt{\frac{\beta_{2}}{\beta_{1}}}
\end{gathered}
$$

where the elements $\Omega_{k 1}^{i}=\psi_{k 1}^{i}$ for $k, l=1, \cdots, 4,(k, l) \neq$ $(1,1), \Omega_{11}^{i}=\left(\psi_{11}^{i}-Z\right)+R_{11} A_{i}+A_{i}^{\mathrm{T}} R_{11}+F_{0} A_{i}+A_{i}^{\mathrm{T}} F_{0}^{\mathrm{T}}$, and we omit $j$ in the calculation of $\beta$.

Note that in general, the results of fuzzy T-S switched systems when applied to only fuzzy systems give large time delay upper bounds because of the absence of the switching modes.

\section{Numerical examples}

In this section, we will present two numerical examples to illustrate the proposed results.

Example 1 ${ }^{[29]}$. Let us consider a non switched system with the following rules

Rule 1: If $x_{1}(t)$ is $W_{1}$, then

$$
\dot{x}=A_{1} x(t)+B_{1} x(t-h) .
$$

Rule 2: If $x_{1}(t)$ is $W_{2}$, then

$$
\dot{x}=A_{2} x(t)+B_{2} x(t-h) .
$$

And the membership functions for Rules 1 and 2 are

$$
\begin{aligned}
& \mu_{1}\left(x_{1}(t)\right)=\frac{1}{1+\mathrm{e}^{-2 x_{1}(t)}} \\
& \mu_{2}\left(x_{2}(t)\right)=1-\mu_{1}\left(x_{1}(t)\right)
\end{aligned}
$$

where $A_{i}$ and $B_{i}(i=1,2)$ are given by

$$
\begin{array}{ll}
A_{1}=\left[\begin{array}{cc}
-2 & 0 \\
0 & -0.9
\end{array}\right], A_{2}=\left[\begin{array}{cc}
-1 & 0.5 \\
0 & -1
\end{array}\right], \\
B_{1}=\left[\begin{array}{cc}
-1 & 0 \\
-1 & -1
\end{array}\right], \quad B_{2}=\left[\begin{array}{cc}
-1 & 0 \\
0.1 & -1
\end{array}\right] .
\end{array}
$$

Taking $\alpha=0$ and applying Corollary 1, we obtain the maximum allowable delay bound $\bar{h}=1.9110$.

To compare with the literature results, the upper bounds on the time delay obtained are listed in Table 1 . It is clear that the obtained upper $\bar{h}$ is significantly larger than those in $[29-35]$.

Table 1 The maximal allowable time delay bound

\begin{tabular}{cc}
\hline Paper & Upper bound \\
\hline Li et al. ${ }^{[31]}$ & 1.00 \\
Tian and Peng & 1.5974 \\
Chen et al. ${ }^{[33]}$ & 1.5974 \\
Wu and Li ${ }^{[34]}$ Corollary 1$)^{[35]}$ & 1.5974 \\
Lien et al. & 1.5974 \\
Peng et al. ${ }^{[29]}$ & 1.6341 \\
Idrissi and Tissir & \\
Corollary 1 & 1.8191 \\
\hline
\end{tabular}

Example 2. Consider continuous switched time-delay system (1) with two subsystems.

For the subsystem 1 ,

Rule 1: If $x_{1}(t)$ is $W_{1}$, then

$$
\dot{x}=\left(A_{11}+\Delta A_{11}(t)\right) x(t)+\left(B_{11}+\Delta B_{11}(t)\right) x(t-h) .
$$


Rule 2: If $x_{1}(t)$ is $W_{2}$, then

$$
\dot{t}=\left(A_{21}+\Delta A_{21}(t)\right) x(t)+\left(B_{21}+\Delta B_{21}(t)\right) x(t-h) .
$$

For the subsystem 2,

Rule 1: If $x_{1}(t)$ is $W_{1}$, then

$\dot{x}(t)=\left(A_{12}+\Delta A_{12}(t)\right) x(t)+\left(B_{12}+\Delta B_{12}(t)\right) x(t-h)$.

Rule 2: If $x_{1}(t)$ is $W_{2}$, then

$\dot{x}(t)=\left(\left(A_{22}+\Delta A_{22}(t)\right) x(t)+\left(B_{22}+\Delta B_{22}(t)\right) x(t-h)\right.$.

And the membership functions are taken the same as in Example 1. The system matrices are given by

$$
\begin{aligned}
& A_{11}=\left(\begin{array}{cc}
-6 & 0.2 \\
-1.05 & 1
\end{array}\right), A_{12}=\left(\begin{array}{cc}
-0.2 & 0.5 \\
-1 & -5
\end{array}\right) \\
& A_{21}=\left(\begin{array}{cc}
-3.6 & -0.3 \\
-0.2 & 0.3
\end{array}\right), A_{22}=\left(\begin{array}{cc}
-0.2 & -0.5 \\
0.31 & -4.9
\end{array}\right) \\
& B_{11}=\left(\begin{array}{cc}
-3 & 0.5 \\
-1 & 1.5
\end{array}\right), B_{12}=\left(\begin{array}{cc}
-0.4 & 0.5 \\
-0.1 & -1
\end{array}\right) \\
& B_{21}\left(\begin{array}{cc}
-0.1 & 0.15 \\
-1 & 0.1
\end{array}\right), B_{22}=\left(\begin{array}{cc}
-0.12 & -0.5 \\
-0.3 & 0.5
\end{array}\right), \\
& H_{a 11}\left(\begin{array}{cc}
-0.01 & 0.0002 \\
-0 & 0.00003
\end{array}\right), H_{a 21}=\left(\begin{array}{cc}
-0.01 & 0 \\
0.002 & 0
\end{array}\right) \\
& H_{a 12}=\left(\begin{array}{cc}
0.01 & 0.0002 \\
0 & -0.00003
\end{array}\right), H_{a 22}=\left(\begin{array}{cc}
-0.0001 & 0 \\
0.0002 & 0
\end{array}\right) \\
& H_{b 11}=\left(\begin{array}{cc}
0.01 & 0 \\
0 & 0.5
\end{array}\right), H_{b 21}=\left(\begin{array}{cc}
0.01 & 0.02 \\
0.25 & 0.3
\end{array}\right), \\
& H_{b 12}=\left(\begin{array}{cc}
0.01 & 0 \\
0.15 & 0.5
\end{array}\right), H_{b 22}=\left(\begin{array}{cc}
0.01 & 0.02 \\
0.25 & 0.3
\end{array}\right), \\
& E_{a 11}=\left(\begin{array}{cc}
0 & 1 \\
0 & -2
\end{array}\right), E_{a 21}=\left(\begin{array}{cc}
2 & 0 \\
0 & 1
\end{array}\right), \\
& E_{a 12}=\left(\begin{array}{cc}
0 & 1 \\
0 & -2
\end{array}\right), E_{a 22}=\left(\begin{array}{cc}
1 & 0 \\
0 & 2
\end{array}\right), \\
& E_{b 11}=\left(\begin{array}{cc}
0.3 & 0 \\
2.2 & 0.3
\end{array}\right), E_{b 21}=\left(\begin{array}{cc}
0.1 & 0.2 \\
0.3 & 0.4
\end{array}\right) \\
& E_{b 12}=\left(\begin{array}{cc}
0.2 & 0 \\
2.2 & 0.3
\end{array}\right), E_{b 22}=\left(\begin{array}{cc}
0.1 & 2 \\
0.3 & 0.4
\end{array}\right) \\
& \left.F_{a 11} t\right)
\end{aligned}
$$

Letting $\alpha=0.5$ and applying Theorem 1, the upper bound $\bar{h}=0.3863$ of $h$ is computed.

\section{Conclusions}

This paper has proposed a switching design for the exponential stability of uncertain linear switching fuzzy timedelay systems. Delay-dependent stability conditions are established by using Lyapunov approach. The perturbations considered are norm-bounded and the results are expressed in terms of LMIs. The numerical examples have been provided to demonstrate the effectiveness and applicability of the proposed method.

\section{References}

[1] D. Liberzon. Switching in Systems and Control, Boston, MA: Birkhäuser Boston, 2003.
[2] A. V. Savkin, R. J. Evans. Hybrid Dynamical Systems: Controller and Sensor Switching Problems, Boston, USA: Birkhäuser Boston, 2002.

[3] Z. D. Sun, S. S. Ge. Switched Linear Systems: Control and Design, London, UK: Springer, 2005.

[4] R. A. De Carlo, M. S. Branicky, S. Pettersson, B. Lennartson. Perspectives and results on the stability and stabilizability of hybrid systems. Proceedings of the IEEE, vol. 88, no. 7, pp. 1069-1082, 2000.

[5] V. N. Phat, S. Pairote. Global stabilization of linear periodically time-varying switched systems via matrix inequalities. Journal of Control Theory and Applications, vol. 4, no. 1, pp. 26-31, 2006.

[6] Z. D. Sun, S. S. Ge. Switched Linear Systems: Control and Design, London, UK: Springer, 2005.

[7] G. S. Zhai, H. Lin, P. J. Antsaklis. Quadratic stabilizability of switched linear systems with polytopic uncertainties. International Journal of Control, vol. 76, no. 7, pp. 747-753, 2003.

[8] T. M. Guerra, L. Vermeiren. LMI-based relaxed nonquadratic stabilization conditions for nonlinear systems in the Takagi-Sugenos form. Automatica, vol.40, no. 5, pp. 823-829, 2004.

[9] W. J. Wang, C. H. Sun. Relaxed stability and stabilization conditions for a T-S fuzzy discrete system. Fuzzy Sets and Systems, vol. 156, no. 2, pp. 208-225, 2005.

[10] B. Yang, D. C. Yu, G. Feng, C. Chen. Stabilisation of a class of nonlinear continuous time systems by a fuzzy control approach. IEE Proceedings: Control Theory and Applications, vol. 153, no. 4, pp. 427-436, 2006.

[11] C. S. Ting. Stability analysis and design of TakagiSugeno fuzzy systems. Information Sciences, vol. 176, no. 19 , pp. 2817-2845, 2006.

[12] M. Boumehraz, K. Benmahamed. Switching controller design for nonlinear systems via fuzzy models. Asian Journal of Information Technology, vol. 5, no. 8, pp. 800-808, 2006.

[13] A. Benzaouia, A. El Hajjaji, F. Tadeo. Stabilization of switching Takagi-Sugeno systems by switching Lyapunov function. International Journal of Adaptive Control and Signal Processing, vol. 25, no. 12, pp. 1039-1049, 2011.

[14] A. Benzaouia. Saturated switching systems. Lecture Notes in Control and Information Sciences, vol. 426, 2012.

[15] E. H. Tissir. Exponential stability and guaranteed cost of switching linear systems with mixed time-varying delays. International Scholarly Research Network ISRN Applied Mathematics, vol. 2011, pp. 1-14, 2011.

[16] L. Van Hien. Exponential stability of switched systems with mixed time delays. Applied Mathematical Sciences, vol. 3 , no. 50, pp. 2481-2489, 2009.

[17] X. H. Liu. Stabilization of switched linear systems with mode dependent time-varying delays. Applied Mathematics and Computation, vol. 261, no. 9, pp. 2581-2586, 2010.

[18] F. Y. Gao, S. M. Zhong, X. Z. Gao. Delay dependent stability of a type of linear switching systems with discrete and distributed time delays. Applied Mathematics and Computation, vol. 196, no. 1, pp. 24-39, 2008.

[19] P. Nyamsup. Stability of time varying switched systems with time varying delay. Nonlinear Analysis: Hybrid Systems, vol. 3, no. 4, pp.631-639, 2009.

[20] X. Y. Lou, B. T. Cui. Delay-dependent criteria for robust stability of uncertain switched Hopfield neural networks. International Journal of Automation and Computing, vol. 4, no. 3, pp. 304-314, 2007. 
[21] Z. L. Xia, J. M. Li. Delay dependent $H_{\infty}$ control for TS fuzzy systems based on a switching Lyapunov function. Acta Automatica Sinica, vol. 35, no. 9, pp. 1235-1239, 2009.

[22] X. Ding, L. Shu. Stability analysis for T-S fuzzy delayed switched systems with time varying perturbation, computational intelligence, foundation and applications. In Proceedings of the 9th International FLINS Conference, pp. 288293, 2010.

[23] Z. L. Xia, J. L. Li. $\mathrm{GH}_{2}$ Control for uncertain discrete-time delay fuzzy systems based on switching fuzzy model and piecewise Lyapunov function. International Journal of $\mathrm{Au}-$ tomation and Computing, vol. 6, no. 3, pp. 261-266, 2009.

[24] Z. L. Xia, J. M. Li, J. R. Li. Delay-dependent non-fragile $H_{\infty}$ filtering for uncertain fuzzy systems based on switching fuzzy model and piecewise Lyapunov function. International Journal of Automation and Computing, vol. 7, no. 4, pp. 428-437, 2010.

[25] Y. Q. Yong, J. M. Jiang, T. P. Zhang, Y. Yi, Q. Zhu. Delay-dependent $H_{2} / H_{\infty}$ control for a class of switched T$\mathrm{S}$ fuzzy systems with time-delay. Advanced Materials Research, vol. 204-210, pp. 1197-1202, 2011.

[26] J. S. Chiou, C. J. Wang, C. M., Cheng, C. C. Wang. Analysis and synthesis of switched nonlinear systems using the T-S fuzzy model. Applied Mathematical Modelling, vol. 34 no. 6 , pp. $1467-1481,2010$.

[27] J. Yonema. New delay dependent approach to robust stability and stabilization for Takagi-Sugeno fuzzy time-delay systems. Fuzzy Sets and Systems, vol.158, no. 20, pp. 2225$2337,2007$.

[28] B. Chen, X. P. Liu. Delay-dependent robust $H_{\infty}$ control for T-S fuzzy systems with time delay. IEEE Transactions on Fuzzy Systems, vol. 13, no. 4, pp. 544-556, 2005.

[29] C. Peng, Y. C. Tian, E. G. Tian. Improved delay-dependent robust stabilization conditions of uncertain T-S fuzzy systems with time varying delay. Fuzzy Sets and Systems vol. 159, no. 20, pp. 2713-2729, 2008.

[30] S. Idrissi, E. H. Tissir. Robust stability of T-S fuzzy systems with time-varying delay and polytypic types uncertainty. International Journal of Ecological Economics and Statistics, vol 24, no 1, pp. 17-25, 2012.
[31] C. G. Li, H. J. Wang, X. F. Liao. Delay-dependent robust stability of uncertain fuzzy systems with time varying delays. IEE Proceedings: Control Theory and Applications, vol. 151, no. 4, pp. 417-421, 2004.

[32] E. G. Tian, C. Peng. Delay dependent stability analysis and synthesis of uncertain T-S fuzzy systems with time-varying delay. Fuzzy Sets and Systems, vol. 157, no.4, pp. 544-559, 2006.

[33] B. Chen, X. P. Liu, S. C. Tong. New delay-dependent stabilization conditions of T-S fuzzy systems with constant delay. Fuzzy Sets and Systems, vol. 158, no. 20, pp. 2209-2224, 2007.

[34] H. N. Wu, H. X. Li. New approach to delay-dependent stability analysis and stabilization for continuous-time fuzzy systems with time-varying delay. IEEE Transactions on Fuzzy Systems, vol. 15, no. 3, pp. 482-493, 2007.

[35] C. H. Lien, K. W. Yu, W. D. Chen, Z. L. Wan, Y. J. Chung. Stability criteria for uncertain Takagi-Sugeno fuzzy systems with interval time-varying delay. IET Control Theory and Applications, vol. 1, no. 3, pp. 764-769, 2007.

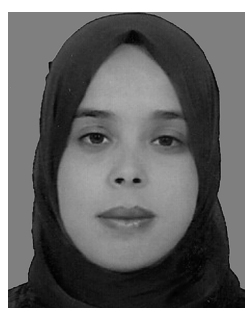

Fatima Ahmida received the M. Sc. degree from Faculty of Sciences, University Sidi Mohammed Ben Abellah, Morocco in 2010.

Her research interests include time delay systems, robust control, switched systems, and fuzzy systems.

E-mail: fatima_ahmida@yahoo.fr

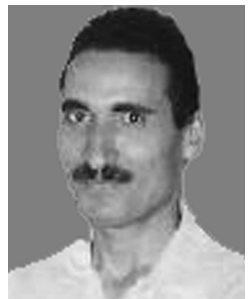

El Houssaine Tissir received the high study diploma (DES) and state doctorate from University Sidi Mohammed Ben Abellah, Faculty of Sciences, Morocco in 1992 and 1997, respectively. He is now a professor at the University Sidi Mohammed Ben Abellah.

His research interests include robust and $H_{\infty}$ control, singular systems, switched and time delay systems, and systems with saturating actuators.

E-mail: elh_tissir@yahoo.fr (Corresponding author) 